\title{
YC-1 [3-(5'-Hydroxymethyl-2'-furyl)-1-benzyl Indazole] Inhibits Neointima Formation in Balloon-Injured Rat Carotid through Suppression of Expressions and Activities of Matrix Metalloproteinases 2 and 9
}

\author{
Yi-Nan Liu, Shiow-Lin Pan, Chieh-Yu Peng, Jih-Hwa Guh, Dong-Ming Huang, \\ Ya-Ling Chang, Chun-Hung Lin, Hui-Chen Pai, Sheng-Chu Kuo, Fang-Yu Lee, and \\ Che-Ming Teng
}

Phamacological Institute (Y.-N.L., S.-L.P., C.-Y.P., D.-M.H., Y.-L.C., C.-H.L., H.-C.P., C.-M.T.), College of Medicine, National Taiwan University, Taipei, Taiwan; School of Pharmacy (J.-H.G.), College of Medicine, National Taiwan University, Taipei, Taiwan; Graduate Institute of Pharmaceutical Chemistry (S.-C.K.), China Medical University, Taichung, Taiwan; and Yung-Shin Pharmaceutical Industry Co., Ltd. (F.-Y.L.), Taichung, Taiwan

Received June 6, 2005; accepted September 21, 2005

\begin{abstract}
Matrix metalloproteinases (MMPs), particularly MMP-2 and MMP-9, and postrevascularization production of vascular smooth muscle cells may play key roles in development of arterial restenosis. We investigated the inhibitory effect of 3-(5'hydroxymethyl-2'-furyl)-1-benzyl indazole (YC-1), a benzyl indazole compound, on MMP-2 and MMP-9 activity in a ballooninjury rat carotid artery model. Injury was induced by inserting a balloon catheter through the common carotid artery; after 14 days, histopathological analysis using immunostaining and Western blotting revealed significant restenosis with neointimal formation that was associated with enhanced protein expression of MMP-2 and MMP-9. However, these effects were dosedependently reduced by orally administered YC-1 (1-10 mg/ $\mathrm{kg}$ ). In addition, gelatin zymography demonstrated that increased MMP-2 and MMP-9 activity was diminished by YC-1
\end{abstract}

treatment. On the other hand, YC-1 inhibited hydrolysis of the fluorogenic quenching substrate Mca-Pro-Leu-Gly-Leu-DpaAla-Arg- $\mathrm{NH}_{2}$ by recombinant MMP-2 and MMP-9 with $\mathrm{IC}_{50}$ values $=2.07$ and $8.20 \mu \mathrm{M}$, respectively. Reverse transcription-polymerase chain reaction analysis of MMP-2 and MMP-9 mRNA revealed that $Y C-1$ significantly inhibited mRNA levels of MMPs. Finally, for the YC-1 treatment group, we did not observe elevation of cGMP levels using enzyme-linked immunosorbent assay, suggesting that YC-1 inhibition of neointimal formation is not through a cGMP-elevating pathway. These data show $\mathrm{YC}-1$ suppression of neointimal formation is dependent on its influence on MMP-2 and MMP-9 protein, mRNA expression, and activity, but not through a cGMP-elevating effect. YC-1 shows therapeutic potential for treatment of restenosis after angioplasty.
During the past 20 years, one focus of cardiovascular pharmaceutical research has been the development of drugs that inhibit intimal hyperplasia. Despite many attempts, no clinical trial has proven that there is an effective pharmacological solution to the problem (Bult, 2000; Garas et al., 2001). Intimal hyperplasia is a late response of the arterial wall to mechanical injury, and it is a major cause of restenosis after

This work was supported by the National Science Council of the Republic of China (Research Grant NSC 94-2811-B-002-017).

Y.-N.L. and S.-L.P. contributed equally to this work.

Article, publication date, and citation information can be found at http://jpet.aspetjournals.org.

doi:10.1124/jpet.105.090563. percutaneous transluminal coronary angioplasty (Lange et al., 1991). After vascular injury, inflammatory cells are recruited to the lesion, where they release several mitogens that activate vascular smooth muscle cells (VSMCs). Consequently, VSMCs are capable of proliferating and migrating from media to intima, releasing matrix and resulting in neointima formation.

Matrix metalloproteinases (MMPs) are a family of structurally related zinc-endopeptidases that degrade components of extracellular matrix associated with vascular remodeling during vascular injury-induced neointima formation (Pauly et al., 1994; Lijnen et al., 1999). MMP-2 and MMP-9, also called gelatinases $\mathrm{A}$ and $\mathrm{B}$, respectively, are the major MMPs

ABBREVIATIONS: VSMC, vascular smooth muscle cell; MMP, matrix metalloproteinase; NO, nitric oxide; sGC, soluble guanylyl cyclase; YC-1, 3-(5'-hydroxymethyl-2'-furyl)-1-benzyl indazole; CMC, carboxymethyl cellulose; ELISA, enzyme-linked immunosorbent assay; GAPDH, glyceraldehyde-3-phosphate dehydrogenase; RT-PCR, reverse transcription-polymerase chain reaction. 
derived from VSMCs and inflammatory cells after vascular injury. Several lines of evidence indicate that MMP-2 and MMP-9 are up-regulated and activated after percutaneous transluminal coronary angioplasty and that they play a key role in regulating VSMC proliferation and migration (Yukihiro et al., 2001). In one study (Bendeck et al., 1994), MMP-9 was expressed within several hours of vascular injury, and MMP-2 activity was markedly increased after 4 days. Based on this hypothesis, MMP inhibitors have been demonstrated to inhibit intimal migration of rat carotid VSMCs in vivo and inhibit subsequent neointima formation (Bendeck et al., 1994; Cho and Reidy, 2002).

Previous research reported that $\mathrm{YC}-1$ is a nitric oxide (NO)-independent, NO-enhancing activator of sGC; it has been used as a research tool for characterization of NO/sGC/ cGMP signaling and function in various tissues (Gomez and Nasi, 2000; Di Fulvio et al., 2001; Suzuki et al., 2001). However, in cardiovascular systems, YC-1 causes an increase in cGMP level and relaxation in vascular smooth muscle (Wegener and Nawrath, 1997; Wegener et al., 1997; Galle et al., 1999). There is also specific heme oxygenase- 1 site overexpression in VSMCs (Imai et al., 2001). This action is a NOindependent, $\mathrm{O}_{2}^{-}$-sensitive activation of sGC in VSMCs. The additive or even synergistic responses to NO-donors of $\mathrm{YC}-1$ in cultured VSMCs and isolated aortic rings apparently reflect the synergistic action of YC-1 and NO on sGC (Mulsch et al., 1997; Galle et al., 1999). On the other hand, YC-1 may inhibit VSMC proliferation to reduce neointima formation through endogenous CO- and/or NO-mediated, cGMP-dependent processes (Wu et al., 1995; Tulis et al., 2000). Despite experimental evidence that YC-1 suppresses intimal hyperplasia in animal models, the effects on MMP-2 and MMP-9 have not been well characterized. In the current work, we studied the in vivo effects of YC-1 on MMP-2 and MMP-9 expression and activity using a carotid artery injury model. Our data show that YC-1 significantly decreased levels of MMP-2 and MMP-9 protein and mRNA and decreased their activities on arterial tissue, almost completely preventing neointimal formation in the injured rat artery.

\section{Materials and Methods}

Materials. YC-1 was supplied by Yung-Shin Pharmaceutical Industry Co., Ltd. (Taichung, Taiwan), anti-rabbit IgG-horseradish peroxidase and anti-mouse IgG-horseradish peroxidase antibody were from Santa Cruz Biotechnology (Santa Cruz, CA), TRIzol reagent was from Invitrogen (Carlsbad, California), random primer and Moloney murine leukemia virus RT were from Promega (Madison, WI), pro Taq was from Protech (Taipei, Taiwan), and antiMMP-2 and anti-MMP-9 antibodies were from Lab Vision (Fremont, California). DAKO LSAB kit was from DAKO (DAKO, Glostrup, Denmark).
Balloon Injury Model. We used the established rat carotid artery (CA) model of balloon angioplasty to examine the in vivo arterial response to injury (Pan et al., 2003). Briefly, male Wistar rats weighing an average of $300 \mathrm{~g}$ were anesthetized with pentobarbital (60 $\mathrm{mg} / \mathrm{kg}$ ), and the left CA was exposed. A Fogarty $2 \mathrm{~F}$ embolectomy balloon catheter was inserted into the left external carotid artery through an arteriotomy incision and advanced to the aortic arch. The balloon was inflated and withdrawn three times with rotation at the same pressure. The catheter was removed, and the external carotid was ligated. The overlying tissue was sutured, and the skin closed with rodent wound clips. Rats were euthanatized by pentobarbital; the left and right (collateral control group) were harvested for study.

YC-1 Dosing. YC-1 suspended in 0.5\% carboxymethyl cellulose (CMC) was orally administered (1, 5, and $10 \mathrm{mg} / \mathrm{kg}$ ) daily to rats beginning from 3 days prior to and lasting for 14 days after balloon injury. In contrast, control animals received only $0.5 \%$ CMC daily. And, the collateral right $\mathrm{CA}$ served as an unmanipulated control.

Tissue Processing. Tissues for enzyme examination were obtained from the sacrificed animals and immediately snap frozen. For each indicated time study examining morphologic remodeling of the vessel wall, carotid arteries were immersion-fixed with $4 \%$ paraformaldehyde in phosphate-buffered saline for $24 \mathrm{~h}$. Then, tissues were transferred to $70 \%$ alcohol and processed by standard procedures and paraffin-embedded. Five-micrometer sections were cut using a rotary microtome and placed on pretreated slides.

Histological Examination and Immunohistochemistry. Histological sections (5 $\mu \mathrm{m}$ in thickness) were stained with hematoxylineosin. Morphometric analysis of cross-sectional areas was performed on arterial sections by using computer-assisted image analysis (NIH Image; National Institutes of Health, Bethesda, MD). Furthermore, sections were immunohistochemically stained for MMP-2 and MMP-9. In brief, 5- $\mu \mathrm{m}$ paraffin sections were deparaffinized, and endogenous peroxidase was destroyed with $0.3 \% \mathrm{H}_{2} \mathrm{O}_{2}$ in $100 \%$ methanol. Antigen was unmasked by $0.1 \%$ trypsin for $10 \mathrm{~min}$ at $37^{\circ} \mathrm{C}$. Tissues were incubated with a monoclonal antibody that recognized the MMP-2 and MMP-9 for $1 \mathrm{~h}$ at room temperature. A standard LSAB technique (DAKO) was used to detect the reaction products.

Zymography. Proteins with gelatinolytic activity were identified by electrophoresis in the presence of sodium dodecyl sulfate (SDS) in $10 \%$ polyacrylamide gels containing $1 \mathrm{mg} / \mathrm{ml}$ gelatin (Mulsch et al., 1997). After sonication, tissue extracts were loaded into gels directly. After electrophoresis, the proteins in the gels were renatured by removing SDS with Triton X-100 (two 15-min incubations in 2.5\% Triton X-100). Subsequently, gels were incubated at $37^{\circ} \mathrm{C}$ in $50 \mu \mathrm{M}$ Tris-HCl, pH 7.4, containing $10 \mu \mathrm{M} \mathrm{CaCl}_{2}$ and $150 \mu \mathrm{M} \mathrm{NaCl}$ for $24 \mathrm{~h}$. At the end of incubation, gels were stained with $0.25 \%$ Coomassie Blue for $1 \mathrm{~h}$. All results were quantified with densitometer.

Measurements of MMP-2 and MMP-9 Activity by EnzymeLinked Immunosorbent Assay. Substrate-linked enzyme-linked immunosorbent assay (ELISA) techniques were used to quantify enzymatic activity of individual MMPs. The samples were thawed on ice, and all reagents needed for the assay were brought to room temperature. The MMP-2 and MMP-9 activity assay (GE Healthcare, Little Chalfont, Buckinghamshire, UK) was performed according to the manufacturer's instructions.

TABLE 1

Synthetic oligonucleotide primers used for RT-PCR

\begin{tabular}{|c|c|c|}
\hline Target Gene & Primer Sequence & Length of PCR Product \\
\hline & & base pairs \\
\hline MMP-2 & $\begin{array}{l}\text { Forward: } 5{ }^{\prime} \text {-ССССТATCTACACCTACACCAAGAAC-3' } \\
\text { Reverse: }\end{array}$ & 576 \\
\hline MMP-9 & $\begin{array}{l}\text { Forward: } 5^{\prime} \text {-AGTTTGGTGTCGCGGAGCAC-3' } \\
\text { Reverse: }\end{array}$ & 755 \\
\hline GAPDH & $\begin{array}{l}\text { Forward: 5'-TGATGACATCAAGAAGGTGGTGAAG-3' } \\
\text { Reverse: } \\
\text { 5'-TCCTTGGAGGCCATGTGGGCCAT-3' }\end{array}$ & 240 \\
\hline
\end{tabular}


Western Blot Analysis. Arterial tissues were homogenized in ice-cold lysis buffer, sonicated, and SDS-polyacrylamide gel electrophoresis was performed on 7.5\% gels for MMP-2 and MMP-9. The blots were electrophoretically transferred to polyvinylidene difluoride membranes and blocked with $5 \%$ nonfat milk for $1 \mathrm{~h}$. Membranes were incubated with either a monoclonal antibody specific for mouse MMP-2 or MMP-9 in phosphate-buffered saline overnight at $4^{\circ} \mathrm{C}$. After incubation with appropriate secondary antibodies, blots were incubated in enhanced chemiluminescence reagents (GE Healthcare) and exposed to photographic film to detect protein expression.

RT-PCR Analysis. RNA was extracted from homogenized tissue with TRIzol reagent by a standard protocol (Invitrogen). Reverse transcription was performed with $5 \mu \mathrm{g}$ of mRNA and random primer at $65^{\circ} \mathrm{C}$ for $5 \mathrm{~min}$ and then mixed with Moloney murine leukemia virus $\mathrm{RT}$ to react at $37^{\circ} \mathrm{C}$ for $1 \mathrm{~h}$ to obtain cDNA. Gene amplification was followed with reverse transcriptase-polymerase chain reaction. Primer sequence was as shown in Table 1. Reaction cycles for MMP-2, MMP-9, and GAPDH include $94^{\circ} \mathrm{C}$ for $5 \mathrm{~min}, 30$ cycles of $94^{\circ} \mathrm{C}$ for $1 \mathrm{~min}, 60^{\circ} \mathrm{C}$ for $1 \mathrm{~min}\left(55^{\circ} \mathrm{C}\right.$ for GAPDH), $72^{\circ} \mathrm{C}$ for $1 \mathrm{~min}$, and a final incubation at $72^{\circ} \mathrm{C}$ for $10 \mathrm{~min}$. PCR products were analyzed on $1.5 \%$ agarose gel in the presence of $1 \mu \mathrm{g} / \mathrm{ml}$ ethidium bromide. The intensities of the cDNA bands were normalized to GAPDH band intensities.

MMP Activities Assay. The enzymatic activity of MMPs was assayed using the fluorogenic substrate Mca-Pro-Leu-Gly-Leu-DpaAla-Arg- $\mathrm{NH}_{2}$. Briefly, samples were mixed with $50 \mathrm{mM}$ HEPES (pH 7.2), $10 \mathrm{mM} \mathrm{CaCl}{ }_{2} \cdot 2 \mathrm{H}_{2} \mathrm{O}, 10 \mu \mathrm{M} \mathrm{ZnCl}_{2}, 0.05 \%$ Brij 35, and dimethyl sulfoxide $(1 \% \mathrm{v} / \mathrm{v})$, containing $2 \mu \mathrm{M}$ substrate. Each assay was carried out at $37^{\circ} \mathrm{C}$ for $3 \mathrm{~h}$, in the dark. After incubation, substrate hydrolysis was measured using spectrofluorimeter, with excitation and emission wavelengths set at 325 and $387 \mathrm{~nm}$, respectively.

Determination of Arterial cGMP Content. All procedures followed manufacturer's instructions (GE Healthcare). Briefly, after sonication, $100-\mu \mathrm{g}$ samples were loaded into a 96 -well cGMP assay plate. Sample competes with cGMP conjugate to the cGMP-binding site on the plate, and then as substrate is injected into plate, substrate interacts with cGMP conjugate to produce blue color. Hence, the more dark blue is the color, the less cGMP is the sample.

Statistical Analysis. Data are presented as the means plus or minus S.E.M. for the indicated number of separate experiments. Statistical analysis of data were performed with one-way analysis of variance followed by a $t$ test, and $P$ values less than 0.05 were considered significant.

\section{Results}

Effect of YC-1 on Neointimal Thickness. The effect of YC-1 on neointimal hyperplasia was quantified by histomorphometric analysis of carotid arterial sections after balloon injury. As shown in Fig. 1, uninjured collateral arteries did not exhibit intimal thickening (Fig. 1A); on the 14th day after balloon injury to the left carotid artery, its neointima was markedly increased (Fig. 1E). Orally administered YC-1 at 1 $\mathrm{mg} / \mathrm{kg} /$ day had a low potency in inhibiting neointima formation; we observed uneven intimal hyperplasia of the vascular wall (Fig. 1F). In contrast, YC-1 at 5 or $10 \mathrm{mg} / \mathrm{kg} /$ day markedly decreased intimal thickening (Fig. 1, G and H). YC-1 had no effect on vascular structure of collateral arteries from the same rats (Fig. 1, B-D).

Effect of YC-1 on MMP-2 and MMP-9 Protein Expression after Injury. To examine the effect of YC-1 on expression of MMP-2 and MMP-9 after balloon injury, vascular sections were stained for specific MMP antibody. Morphometric analysis of common carotid arteries revealed a marked positive staining for both MMP-2 and MMP-9 protein

\section{Uninjured}

Injured

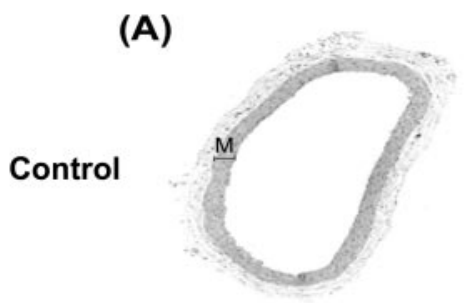

(E)

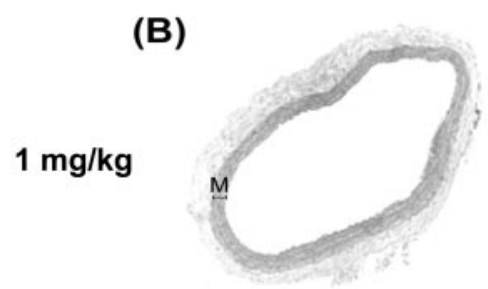

(F)

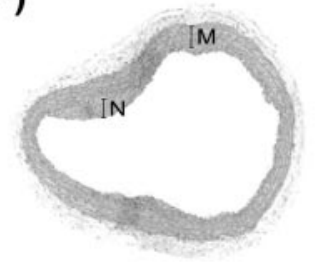

(C)

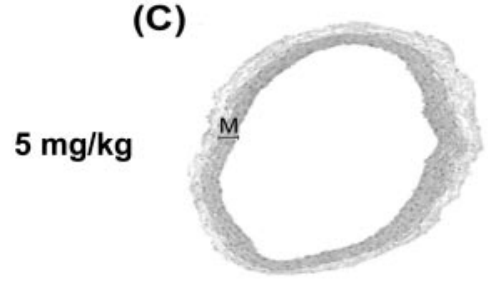

(G)

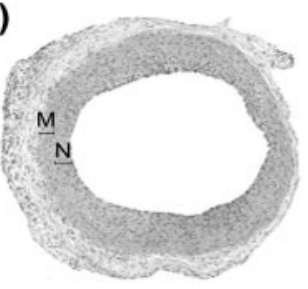

(D)
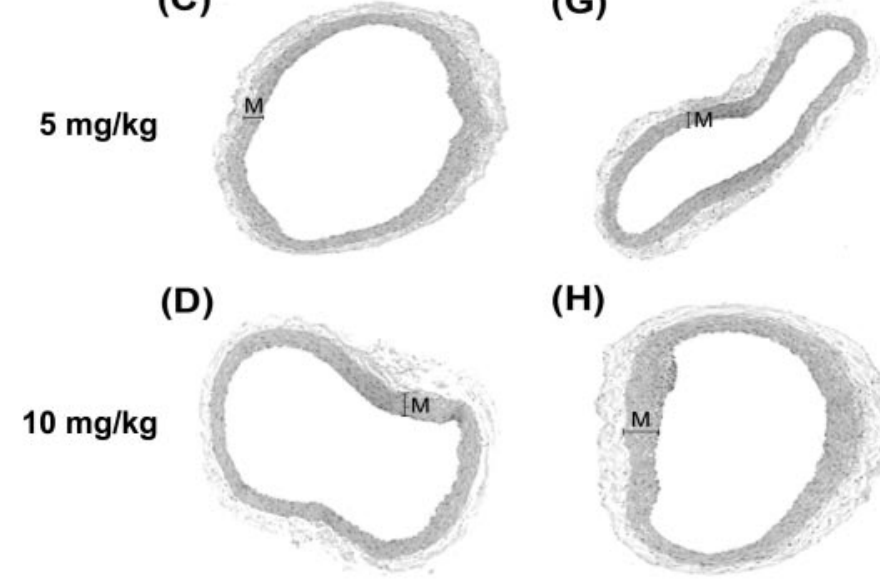

(H)

(I)

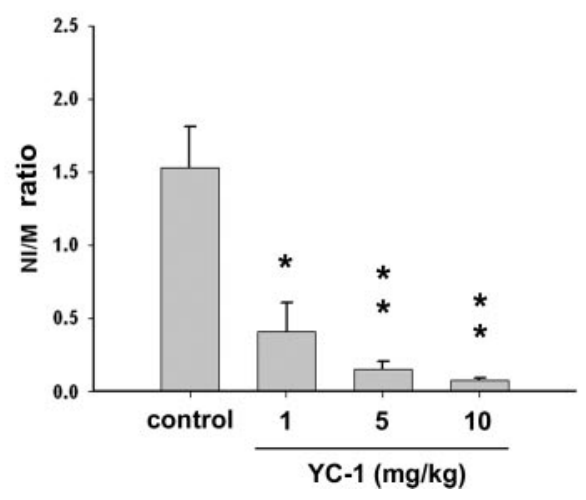

Fig. 1. Effects of YC-1 on the intimal hyperplasia of the carotid arteries 14 days after injury. Hematoxylin-eosin staining sections of carotid arteries from the control (A and E) and YC-1 (1-10 mg/kg)-treated (B-D and $\mathrm{F}-\mathrm{H})$ groups. Magnification for all photomicrographs is $40 \times$. "N" represents neointima, and "M" represents media. I, data are also quantified by the neointima/media ratio of common carotid arteries after balloon injury from each group of animal studies. Data are expressed as mean \pm S.E.M. of six determinations. $*, P<0.05$; and $* *, P<0.01$ compared with control group.

in the intima of injured carotid arteries (Fig. 2, C and E) compared with control arteries (anti-IgG group; Fig. 2A) on the 14th day after balloon injury. However, oral administra- 


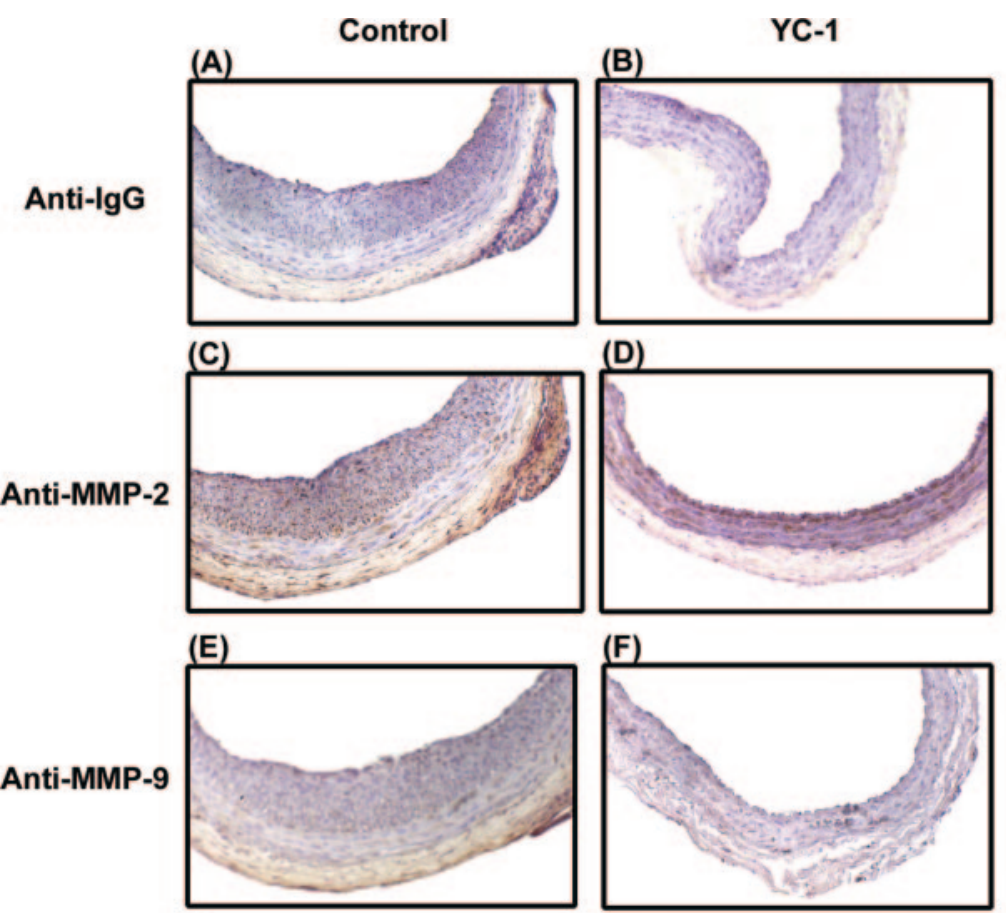

(G)
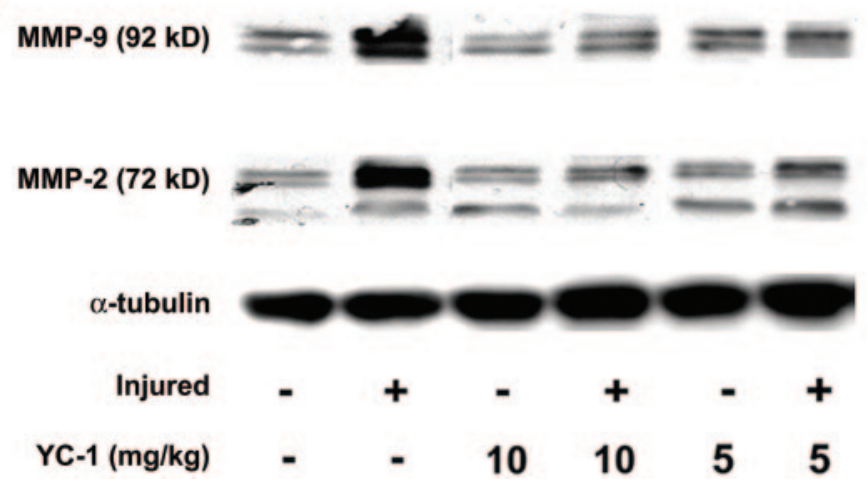

Fig. 2. Effect of YC-1 on expression and location of MMP-2 and MMP-9 after balloon injury. Immunohistochemical staining was performed by MMP-2 and MMP-9 antibody from the control (A, C, and E) and YC-1 (10 mg/kg)-treated $(\mathrm{B}, \mathrm{D}$, and F) groups after balloon-injured carotid arteries, as detailed under Materials and Methods. Staining was performed in $\mathrm{A}$ and $\mathrm{B}, \mathrm{C}$ and $\mathrm{D}$, and $\mathrm{E}$ and $\mathrm{F}$ using antiIgG, anti-MMP-2, and anti-MMP-9 antibody, respectively. Magnification $200 \times$. Representative immunostaining sections are shown from six animals in each treatment group G, MMP-2 and MMP-9 protein expressions were identified by Western blot analysis using anti-MMP2 and antiMMP-9 antibodies. tion of $10 \mathrm{mg} / \mathrm{kg}$ YC-1 significant inhibited MMP-2 and MMP-9 protein expression in carotid artery sections after balloon injury (Fig. 2, D and F). Next, we analyzed artery extracts for MMP-2 and MMP-9 protein expression using Western blot analysis. MMP-2 and MMP-9 protein expression markedly increased in injured artery but were inhibited to baseline with YC-1 treatment of 5 or $10 \mathrm{mg} / \mathrm{kg}$ (Fig. 2G).

Effects of YC-1 on Postinjury MMP Activity by Gelatin Zymography and ELISA Assay. We studied the suppressive effects of YC-1 on MMP-2 and MMP-9 activity in balloon-injured carotid vessels using zymographic analysis and ELISA assay. As shown in Fig. 3, YC-1 (1-10 mg/kg) inhibited injury-evoked MMP-2 and MMP-9 activity in dosedependent manner on the 14th day after balloon injury.

Effects of YC-1 on MMP Activity by Quenched Fluorescence Substrate Hydrolysis. To directly test for inhibition of enzyme activity, we performed a concentration-effect study of YC-1 action on recombinant MMP-2 and MMP-9 activity with use of the fluorescence quenching substrate Mca-Pro-Leu-Gly-Leu-Dpa-Ala-Arg- $\mathrm{NH}_{2}$. Concentration-dependent inhibition of YC-1 on MMP-2 and MMP-9 activity is shown in Table 2. Significant inhibition was demonstrated at YC-1 concentrations as low as $1 \mu \mathrm{M}$; at $10 \mu \mathrm{M}, \mathrm{YC}-1$ almost completely abolished enzyme activity. The $\mathrm{IC}_{50}$ values for YC-1 were 2.07 and 8.20 $\mu \mathrm{M}$ for MMP-2 and MMP-9, respectively.

Effect of YC-1 on MMP mRNA Expression. In addition, we analyzed mRNA expression of MMPs by RT-PCR. Each experiment included a pool of three to six independent animals treated with the same conditions. We observed that levels of both MMP-2 and MMP-9 mRNA were increased on the 14th day after balloon injury and that YC-1 suppressed both but was more effective at inhibiting MMP-9 than MMP-2 (Fig. 4). These data suggest that YC-1 inhibits MMP-2 and -9 protein productions, activities, and mRNA expressions after balloon injury.

Effect of YC-1 on cGMP Content. To evaluate the effect of balloon injury and YC-1 on cGMP formation in rat carotid arteries, sections from injured and uninjured vessels in $0.5 \%$ CMC- or YC-1-treated groups were assayed using a cGMP ELISA kit. After balloon-injured 5th $\mathrm{h}$ and 14 th day time point, we did not observe any elevation of cGMP levels in the presence or absence of YC-1-treated rats (Fig. 5). This result indicates that YC-1 suppressed MMP-2, MMP-9 activity and expression via a cGMP-independent pathway. 
(A)

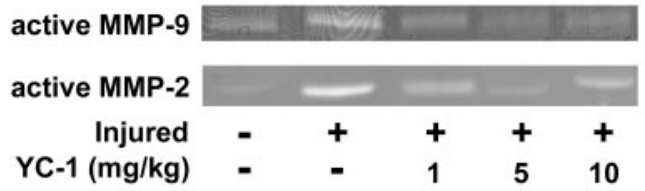

(B)

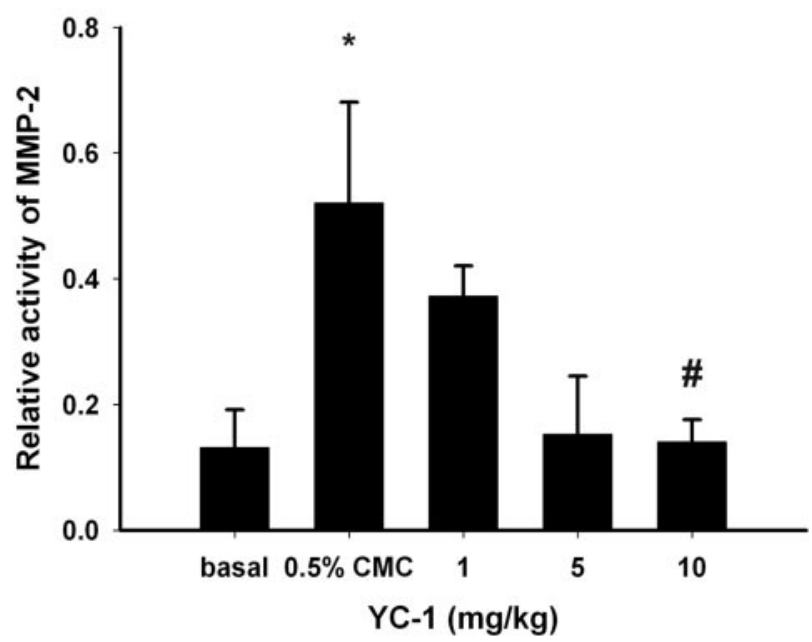

(C)

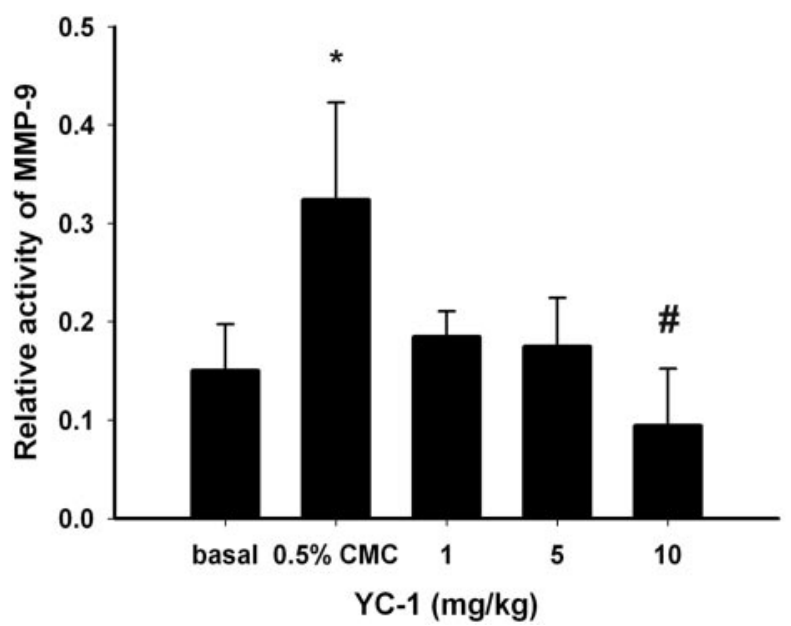

Fig. 3. Effects of YC-1 on MMP-2 and MMP-9 gelatinase activities at 14 days after balloon injury. Arteries were harvested from rats treated with $0.5 \%$ CMC or YC-1 (1, 5, and $10 \mathrm{mg} / \mathrm{kg} /$ day) for 3 days before injury. After injury, rats were treated with the same drug for the following 14 days. Proteins were harvested from injured and uninjured carotid arteries after balloon injury. A, equal amounts of protein $(10 \mu \mathrm{g})$ were resolved on $10 \%$ gelatin zymography gels containing $0.1 \%$ gelatin. Zymograms were scanned, and MMP-2 (B) or MMP-9 (C) activity was quantified. "Basal group", arteries were harvested from uninjured carotid arteries. "Control group", arteries were harvested from injured carotid arteries. *, $P<0.05$ compared with uninjured carotid arteries of rats; and \#, $P<0.05 \mathrm{com}$ pared with injured carotid arteries of rats at 14 days after balloon injury. Data are means \pm S.E.M. for six independent experiments.

\section{Discussion}

In this study, we demonstrated that YC-1, an indazolederived compound, significantly inhibits balloon-injury-induced neointimal formation as well as production of MMP-2
TABLE 2

$\mathrm{IC}_{50}$ values and inhibition for YC-1 on the activity of MMP-2 and MMP-9

Values are given as means \pm S.E.M. $(n=3)$. The $\mathrm{IC}_{50}$ values for YC-1 $(0.01-10 \mu \mathrm{M})$ were determined using the MMP substrate assay. Details are described under Materials and Methods.

\begin{tabular}{crcrrr}
\hline \multirow{2}{*}{ YC-1 Concentration } & \multicolumn{2}{c}{ MMP-2 } & & \multicolumn{2}{c}{ MMP-9 } \\
\cline { 2 - 3 } \cline { 5 - 6 } \cline { 5 - 6 } & Inhibition & $\mathrm{IC}_{50}$ & & Inhibition & $\mathrm{IC}_{50}$ \\
\hline$\mu M$ & $\%$ & $\mu M$ & & $\%$ & $\mu M$ \\
10 & 102 & & & 59 & \\
1 & 18 & $2.07 \pm 0.11$ & & 3 & $8.20 \pm 0.34$ \\
0.1 & 4 & & & 2 & \\
0.01 & 2 & & & 0 & \\
\hline
\end{tabular}

and MMP-9, two proteins that have been implicated in intimal matrix breakdown. Proliferation and migration of VSMCs play a major role in intimal lesion formation (Liu et al., 1989; Pauletto et al., 1994). In previous studies, YC-1 has been shown to significantly inhibit VSMC proliferation through a cGMP-dependent pathway (Tulis et al., 2000; Wu et al., 2004) and to inhibit expression of transforming growth factor- $\beta$ and focal adhesion kinase (Wu et al., 2004). However, all of these experiments have used topical administration of YC-1 to study inhibition of neointimal formation after balloon-induced carotid artery injury. In this study, we demonstrated that low-dose oral administration $(1-10 \mathrm{mg} / \mathrm{kg})$ of YC-1 markedly prevents neointimal formation. Based on pharmacokinetic analysis, this dose range does not induce sGC activity (S. C. Kuo, F. Y. Lee, and C. M. Teng, unpublished data); for that to happen, the dose must be higher than $100 \mathrm{mg} / \mathrm{kg}$. On the other hand, we evaluated the effect of balloon injury and YC-1 on cGMP formation in rat carotid arteries and did not find an increased level of cGMP; this observation is in contrast to previous reports (Tulis et al., 2000,2002 ). We also did not observe elevation of cGMP levels in YC-1-treated rats, suggesting that the therapeutic effect of YC-1 on restenosis may not involve a cGMP-elevating pathway.

It is well established that MMPs play important roles in mediating intimal thickening after vascular injury (Galis and Khatri, 2002; Kuzuya and Iguchi, 2003). MMPs, important zinc-dependent proteinases responsible for matrix degradation, are involved in VSMC proliferation and migration, two key steps in intimal hyperplasia (Galis and Khatri, 2002; Kuzuya and Iguchi, 2003). Southgate et al. (1996) also demonstrated that MMP-2 and MMP-9 mRNA expression increased markedly following vascular injury and that the proteinases played a role in migration and proliferation of VSMCs. Consistent with these latter observations, we observed marked thickening characteristic of neointimal formation on the 14th day after balloon injury. MMP-2 and MMP-9 clearly participated in neointima formation based on our demonstration that both the activity and protein expression of MMP-2 and MMP-9 increased almost at the same time. In the present study, we demonstrated that YC-1 inhibited MMP activity as well as protein and mRNA expression. On the other hand, our data revealed that YC-1 could suppress MMP-2 and MMP-9 enzyme activity with an $\mathrm{IC}_{50}$ value in the micromolar range. As far as we know, this is the first report to show that YC-1 is capable of inhibiting MMPs after balloon injury in rats.

Increased MMP activity is associated with a wide variety of 
(A)

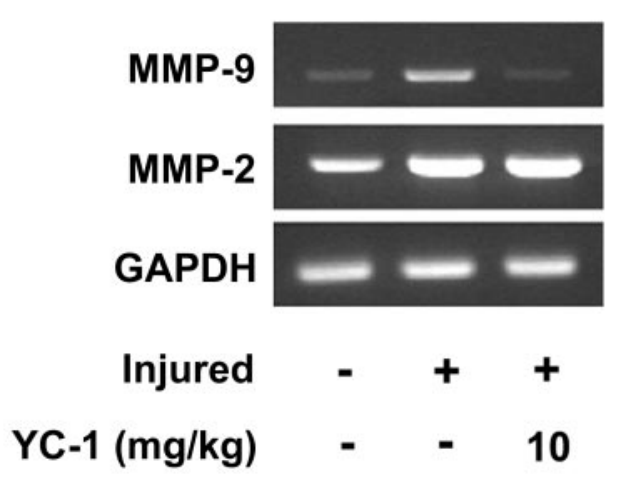

(B)

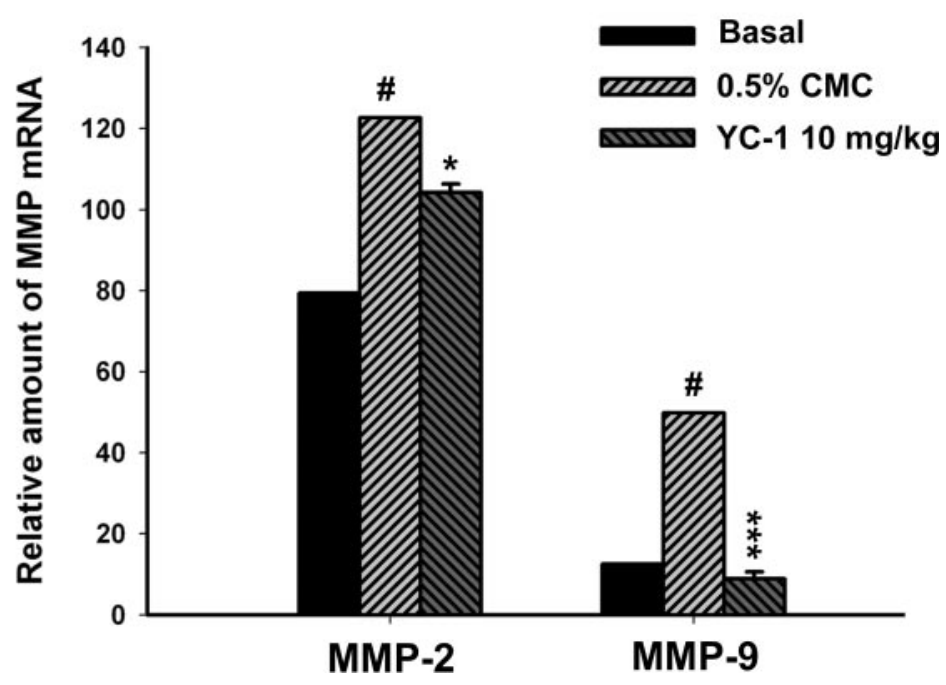

Fig. 4. Effects of YC-1 on MMP-2 and MMP-9 mRNA expression 14 days after balloon injury. A, MMP-2 and MMP-9 mRNA expression by RT-PCR and (B) quantified by densitometry. "Basal", arteries were harvested from uninjured carotid arteries. Data are presented as mean \pm S.E.M. Each result is from two to four independent experiments, and each experiment includes mean of three to six independent animals in panel. \#, $P<0.05$ compared with uninjured carotid arteries of rats, and $*, P<0.05$; ***, $P<$ 0.001 compared with injured carotid arteries of rats 14 days after balloon injury.

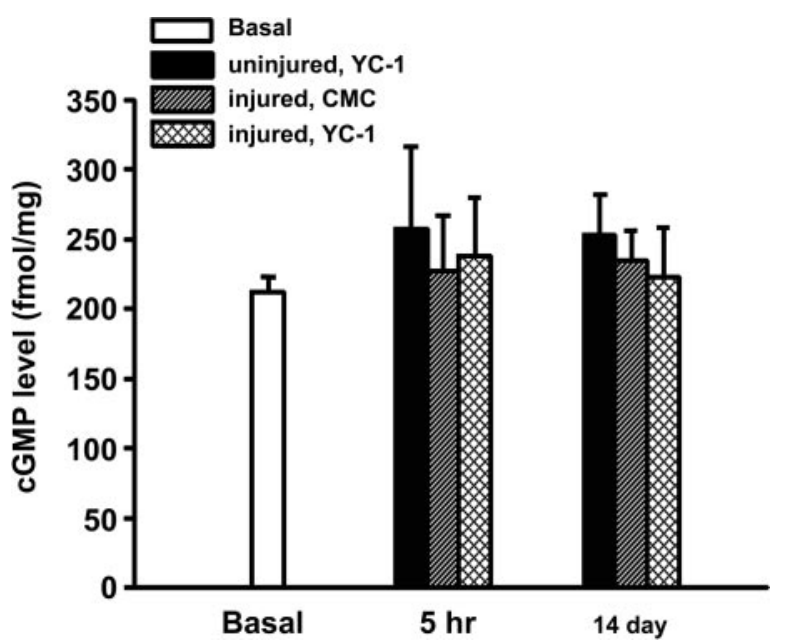

Fig. 5. Effect of YC-1 on cGMP formation after balloon injury. Arteries were harvested at 5 th $\mathrm{h}$ or 14 th day after balloon injury. Rats were treated with $0.5 \% \mathrm{CMC}$ or YC-1 $10 \mathrm{mg} / \mathrm{kg} /$ day, respectively. Data are presented as mean \pm S.E.M. Different time points are from six independent animals.

pathological conditions, such as restenosis and atherosclerosis (Galis and Khatri, 2002; Kuzuya and Iguchi, 2003). The potential utility of MMP inhibitors as treatment for these diverse disease states is obvious, and several MMP inhibitors are currently being tested in clinical trials (Ramnath and Creaven, 2004). However, undesirable side effects have greatly limited clinical use to date (Overall and Lopez-Otin, 2002). Several recent studies have suggested that MMPmediated vascular remodeling in response to inflammatory effects could be modulated by antioxidants (Galis et al., 1998). Our recent study has shown that YC-1 is capable of inhibiting lipopolysaccharide-induced inducible nitric-oxide synthase, tumor necrosis factor- $\alpha$, and cytokine release through down-regulation of nuclear factor- $\kappa \mathrm{B}$ activity (Pan et al., 2005). It is possible that YC-1 down-regulates MMP-2 and MMP-9 via diminished transactivation because the $5^{\prime}$ flanking region of rat MMP genes contain binding sequences for the nuclear factor- $\kappa \mathrm{B}$-transactivating molecule. Hence, YC-1 may inhibit MMP activation through a decreased inflammatory response, and this inspection needs more investigation. Moreover, we demonstrated that YC-1 inhibited MMP expression on the 14th day after balloon injury. This late phase was prone to a response of vascular VSMC proliferation and matrix secretion, but it was independent of the early postinjury inflammatory response. We demonstrated that MMP-2 and MMP-9 mRNA were up-regulated on the 14th day after balloon injury and that YC-1 was capable of inhibiting MMP mRNA up-regulation as well as other known MMP inhibitors. We conclude that YC-1 inhibits MMP activity through inhibition of late-phase VSMC proliferation with 
the result of suppressing neointimal hyperplasia. In summary, we clearly demonstrated in vivo that oral administration of YC-1 inhibits neointima formation and MMP activation after balloon injury to the carotid artery. Based on these observations, YC-1 shows therapeutic potential for prevention of restenosis after angioplasty.

\section{References}

Bendeck MP, Zempo N, Clowes AW, Galardy RE, and Reidy MA (1994) Smooth muscle cell migration and matrix metalloproteinase expression after arterial injury in the rat. Circ Res 75:539-545.

Bult H (2000) Restenosis: a challenge for pharmacology. Trends Pharmacol Sci 21:274-279.

Cho A and Reidy MA (2002) Matrix metalloproteinase-9 is necessary for the regulation of smooth muscle cell replication and migration after arterial injury. Circ Res 91:845-851.

Di Fulvio M, Lauf PK, and Adragna NC (2001) Nitric oxide signaling pathway regulates potassium chloride cotransporter-1 mRNA expression in vascular smooth muscle cells. J Biol Chem 276:44534-44540.

Galis ZS, Asanuma K, Godin D, and Meng X (1998) $N$-Acetyl-cysteine decreases the matrix-degrading capacity of macrophage-derived foam cells: new target for antioxidant therapy? Circulation 97:2445-2453.

Galis ZS and Khatri JJ (2002) Matrix metalloproteinases in vascular remodeling and atherogenesis: the good, the bad and the ugly. Circ Res 90:251-262.

Galle J, Zabel U, Hubner U, Hatzelmann A, Wagner B, Wanner C, and Schmidt HH (1999) Effects of the soluble guanylyl cyclase activator, YC-1, on vascular tone, cyclic GMP levels and phosphodiesterase activity. Br J Pharmacol 127:195-203.

Garas SM, Huber P, and Scott NA (2001) Overview of therapies for prevention of restenosis after coronary interventions. Pharmacol Ther 92:165-178.

Gomez MP and Nasi E (2000) Light transduction in invertebrate hyperpolarizing photoreceptors: possible involvement of a Go-regulated guanylate cyclase. J Neurosci 20:5254-5263.

Imai T, Morita T, Shindo T, Nagai R, Yazaki Y, Kurihara H, Suematsu M, and Katayama S (2001) Vascular smooth muscle cell-directed overexpression of heme oxygenase-1 elevates blood pressure through attenuation of nitric oxide-induced vasodilation in mice. Circ Res 89:55-62.

Kuzuya M and Iguchi A (2003) Role of matrix metalloproteinases in vascular remodeling. J Atheroscler Thromb 10:275-282.

Lange RA, Flores ED, and Hillis LD (1991) Restenosis after coronary balloon angioplasty. Annu Rev Med 42:127-132.

Lijnen HR, Lupu F, Moons L, Carmeliet P, Goulding D, and Collen D (1999) Temporal and topographic matrix metalloproteinase expression after vascular injury in mice. Thromb Haemost 81:799-807.

Liu MW, Roubin GS, and King SB 3rd (1989) Restenosis after coronary angioplasty. Circulation 79:1374-1387.

Mulsch A, Bauersachs J, Schafer A, Stasch JP, Kast R, and Busse R (1997) Effect of YC-1, an NO-independent, superoxide-sensitive stimulator of soluble guanylyl cyclase, on smooth muscle responsiveness to nitrovasodilators. $\mathrm{Br}$ J Pharmacol 120:681-689.
Overall CM and Lopez-Otin C (2002) Strategies for MMP inhibition in cancer: innovations for the post-trial era. Nat Rev Cancer 2:657-672.

Pan SL, Guh JH, Peng CY, Chang YL, Cheng FC, Chang JH, Kuo SC, Lee FY, and Teng CM (2005) A potential role of YC-1 on the inhibition of cytokine release in peripheral blood mononuclear leukocytes and endotoxemic mouse models. Thromb Heamost 93:940-948.

Pan SL, Huang YW, Guh JH, Chang YL, Peng CY, and Teng CM (2003) Esculetin inhibits Ras-mediated cell proliferation and attenuates vascular restenosis following angioplasty in rats. Biochem Pharmacol 65:1897-1905.

Pauletto P, Sartore S, and Pessina AC (1994) Smooth-muscle-cell proliferation and differentiation in neointima formation and vascular restenosis. Clin Sci 87:467479.

Pauly RR, Passaniti A, Bilato C, Monticone R, Cheng L, Papadopoulos N, Gluzband YA, Smith L, Weinstein C, and Lakatta EG (1994) Migration of cultured vascular smooth muscle cells through a basement membrane barrier requires type IV collagenase activity and is inhibited by cellular differentiation. Circ Res 75:41-54

Ramnath N and Creaven PJ (2004) Matrix metalloproteinase inhibitors. Curr Oncol Rep 6:96-102.

Southgate KM, Fisher M, Banning AP, Thurston VJ, Baker AH, Fabunmi RP Groves PH, Davies M, and Newby AC (1996) Upregulation of basement membrane-degrading metalloproteinase secretion after balloon injury of pig carotid arteries. Circ Res 79:1177-1187.

Suzuki T, Suematsu M, and Makino R (2001) Organic phosphates as a new class of soluble guanylate cyclase inhibitors. FEBS Lett 507:49-53.

Tulis DA, Bohl Masters KS, Lipke EA, Schiesser RL, Evans AJ, Peyton KJ, Durante W, West JL, and Schafer AI (2002) YC-1-mediated vascular protection through inhibition of smooth muscle cell proliferation and platelet function. Biochem Biophys Res Commun 291:1014-1021.

Tulis DA, Durante W, Peyton KJ, Chapman GB, Evans AJ, and Schafer AI (2000) YC-1, a benzyl indazole derivative, stimulates vascular cGMP and inhibits neointima formation. Biochem Biophys Res Commun 279:646-652.

Wegener JW, Gath I, Forstermann U, and Nawrath H (1997) Activation of soluble guanylyl cyclase by YC-1 in aortic smooth muscle but not in ventricular myocardium from rat. $\mathrm{Br} J$ Pharmacol 122:1523-1529.

Wegener JW and Nawrath H (1997) Differential effects of isoliquiritigenin and YC-1 in rat aortic smooth muscle. Eur J Pharmacol 323:89-91.

Wu CC, Ko FN, Kuo SC, Lee FY, and Teng CM (1995) YC-1 inhibited human platelet aggregation through NO-independent activation of soluble guanylate cyclase. $\mathrm{Br}$ Pharmacol 116:1973-1978.

Wu CH, Chang WC, Chang GY, Kuo SC, and Teng CM (2004) The inhibitory mechanism of YC-1, a benzyl indazole, on smooth muscle cell proliferation: an in vitro and in vivo study. J Pharmacol Sci 94:252-260.

Yukihiro H, Ikeda U, Katsuki T, Mizuno O, Fujikawa H, and Shimada K (2001) Matrix metalloproteinase expression in the coronary circulation induced by coronary angioplasty. Atherosclerosis 161:185-192.

Address correspondence to: Dr. Che-Ming Teng, Pharmacological Institute College of Medicine, National Taiwan University, 1, Jen-Ai Road, Sect. 1, Taipei, Taiwan. E-mail: cmteng@ha.mc.ntu.edu.tw 